\title{
Is the obesity a risk factor for delayed colonic post-polypectomy bleeding?-authors' reply
}

\author{
Veeravich Jaruvongvanich ${ }^{1}$
}

Accepted: 12 February 2018 / Published online: 21 February 2018

(C) Springer-Verlag GmbH Germany, part of Springer Nature 2018

\section{Dear Editor:}

We highly appreciate the comments from Coskun [1] as to whether obesity could be a risk factor for delayed colonic postpolypectomy bleeding (PPB). We conducted a meta-analysis, which identified cardiovascular disease, hypertension, polyp size, and polyp location as risk factors for PPB [2]. The author highlighted that body mass index (BMI) is defined as a risk factor for delayed endoscopic submucosal dissection bleeding. Since obesity is a global epidemic and is highly prevalent, this factor must be taken into consideration.

We reviewed the 12 included studies of our meta-analysis. Only 3 studies investigated BMI in their studies. Two studies (Kim et al. and $\mathrm{Wu}$ et al.) compared the mean BMI between patients with and without $\mathrm{PPB}$ and showed a non-significantly higher BMI in patients with PPB than those without PPB. Kwon et al. conducted a multivariate analysis adjusted for tumor location, shape, and size and found 3.6 times higher odds of PPB in patients with BMI $>25 \mathrm{~kg} / \mathrm{m}^{2}$ than those with $\mathrm{BMI}<25 \mathrm{~kg} / \mathrm{m}^{2}$. All 3 studies were case-control studies. There were too few studies to conduct a meta-analysis. Kim et al. and Wu et al. found that the BMI was numerically higher among patients who developed PPB although the result did not reach statistical significance, which could be due to the fact that the studies were too small (number of subjects were 120 and 210). However, the majority of studies did not report or assess obesity as a possible risk. We further searched with the same search criteria as our original study until January 31, 2018, and found no further studies that address this question. Further prospective studies regarding the risk factors for colonoscopic PPB should investigate obesity as part of the possible risk factors.

\section{References}

1. Coskun AK (2017) Is the obesity a risk factor for delayed colonic post-polypectomy bleeding? Int J Color Dis 32:1661

2. Jaruvongvanich V, Prasitlumkum N, Assavapongpaiboon B, Suchartlikitwong S, Sanguankeo A, Upala S (2017) Risk factors for delayed colonic post-polypectomy bleeding: a systematic review and meta-analysis. Int J Color Dis 32:1399-1406
Veeravich Jaruvongvanich veeravich_j@hotmail.com

1 Internal Medicine, University of Hawaii, Honolulu, HI, USA 